\title{
The Analysis and Control for Singular Ecological-Economic Model with Harvesting and Migration
}

\author{
Qingling Zhang, ${ }^{1}$ Hong Niu, ${ }^{1}$ Lichun $\mathrm{Zhao}^{2}{ }^{2}$ and Fenglan Bai ${ }^{3}$ \\ ${ }^{1}$ Institute of Systems Science, Northeastern University, Liaoning, Shenyang 110819, China \\ 2 Department of Mathematics, Anshan Normal University, Liaoning, Anshan 114007, China \\ ${ }^{3}$ School of Science, Dalian Jiaotong University, Liaoning, Dalian 116028, China \\ Correspondence should be addressed to Qingling Zhang, qlzhang@mail.neu.edu.cn
}

Received 12 March 2012; Accepted 11 April 2012

Academic Editor: Zhiwei Gao

Copyright (c) 2012 Qingling Zhang et al. This is an open access article distributed under the Creative Commons Attribution License, which permits unrestricted use, distribution, and reproduction in any medium, provided the original work is properly cited.

To keep the resources renewable, a singular ecological-economic model is proposed for the populations with harvesting and migration. The local stability and the dynamic behavior of the model are studied. Singular induced bifurcation appears when economic interest is zero, which is different from the ordinary differential models. In order to apply variable structure control to eliminate these complex behaviors, the singular model is transformed into a single-input and single-output model with parameter varying within definite intervals. And then, a variable structure controller is designed to make the model stable. Finally, an inshore-offshore fishery model is given to illustrate the proposed method, and some numerical simulations are shown to demonstrate the control results.

\section{Introduction}

The management of renewable resources is important for the development of human and society. In exploiting the biological resources, both the economic profit and the environmental effects should be taken into account, which initiates a new research area: biomathematics. Interactions of mathematics and biology promote the development of the biosciences greatly in a certain extent. Since most of biological theories evolve rapidly, it is necessary to develop some useful mathematical models to describe the consequences of these biological models.

Singular model as a branch of modern control theory can describe a class of practical models more accurately. Compared with the ordinary differential models, singular models exhibit more complicated dynamics, such as the impulse phenomenon. They have more applications in power systems, aerospace engineering, chemical processes, social economic systems, biological systems, network analysis, and so forth. With the help of the singular 
models for the power systems and bifurcation theory, complex dynamical behaviors of the power systems have been extensively studied, which reveal the instability mechanism of power systems [1-3]. Applications of singular models are also found in neural networks [4], fault diagnosis $[5,6]$, robotics $[7,8]$ and epidemic [9-11], economics $[12,13]$, and chemistry [14]. As far as the singular system theory is concerned, there are a few research results in biology. Since a singular biological economics model with stage structure was established to model the biological systems in [15], some singular biological models appeared [16-19]. These ideas are based on the economic theory [20]:

Net Economic Revenue $=$ Total Revenue - Total Cost.

This formula presents some solid preliminary on singular biological systems.

In biology, many mathematicians, ecologists, and economists are concerned with the exploitation of renewable resources in recent years, and some results are achieved [21-24]. Though the harvesting can bring economic profit for people, the overexploitation may cause the extinction of some populations. In order to prevent the population from damages, some methods are introduced, such as, to raise taxes or to make the young population forbidden to be harvested. We propose a singular ecological-economic model to model such a problem. Singular model is often strongly nonlinear and unstable. In this case, one of control methods, which are able to perform high-quality automatic control, is demanded.

Variable structure control is considered to be used in this paper. It is a flexible control method to deal with some models with uncertain parameters and external disturbances. The main advantage of this technique is that once the system state variables reach a sliding surface, the structure of the feedback loop is adaptively altered to slide the state variables along the sliding surface. Thereafter, the system response depends on the gradients of the sliding surface and remains insensitive to parameter variations and external disturbances. Variable structure control with sliding mode was first proposed by Emelyanov [25] and was elaborated in the 1970s $[26,27]$. In their pioneer works, variable structure controls are used to handle some linear models, and then expanded to nonlinear models, multi-input and multioutput models, discrete time models, infinite-dimensional models and stochastic models [28-33]. In recent years, variable structure control is applied to a wide variety of engineering fields successfully, such as robot control, flight control, motor control, and power control [34-36].

The main contents of the paper are as follows. In Section 1, in order to prevent the extinction of some populations, a singular ecological-economic model is proposed for the populations with harvesting and migration. In Section 2, when the local stability and the dynamic behavior for the model are discussed, singular induced bifurcation appears, and a control method is demanded to eliminate this bifurcation. In Section 3, in order to apply variable structure control, the singular model is transformed into a single-input and singleoutput model with parameters varying within definite intervals. In Section 4, an inshoreoffshore model is given to illustrate the analysis results, and the simulations illustrate the effectiveness of the proposed method.

\section{Modeling}

In order to model growth of the populations, numerous models have been introduced. The generalized logistic growth model can provide an adequate approximation for the growth 


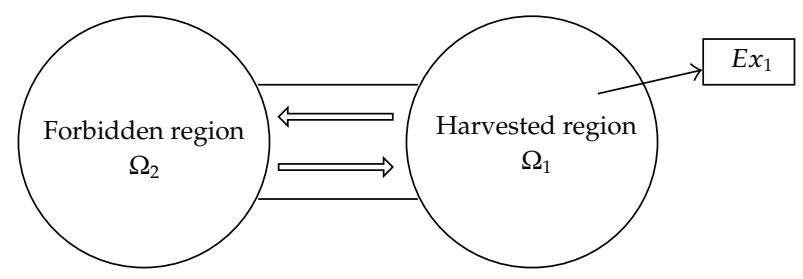

Figure 1: The migration between the harvesting permitted region and the harvesting forbidden region.

of the populations. However, if there is no harvesting, the populations would continue to increase rapidly. Therefore, the harvesting is an effective measure to maintain the diversity of species and protect the renewable resources. The equation of the harvested populations reads

$$
\frac{d x}{d t}=\gamma x\left(1-\frac{x}{K}\right)-q E x
$$

where $x$ is the number of population, $\gamma$ is a positive constant which is called the intrinsic growth rate, $E$ is the harvesting effort, $q$ is catch-ability coefficient, and $K$ is usually the environment carrying capacity or saturation level. Some papers studied the model (2.1) with a constant effort $E$. But it is only suitable for some special case. In practice, the harvesting effort $E$ is usually time-varying. For convenience in calculation, the condition $q=1$ is usually assumed. If $E>\gamma$, a rapid collapse of the populations will occur. The extinction of population is inevitable, and the ecological balance will be destroyed.

In order to keep the resources renewable and prevent the extinction of some populations, the populations can be divided into two regions: $\Omega_{1}$ and $\Omega_{2}$. In region $\Omega_{1}$, the harvesting is permitted, while the harvesting is forbidden in region $\Omega_{2}$. If there is a difference between region $\Omega_{1}$ and region $\Omega_{2}$, the migration can occur between two regions, which is assumed to be proportional to the difference, and the proportional coefficient is positive. To better understand relation of the populations between two regions, a concise schematic is shown in Figure 1.

From Figure 1, it can be seen that the two regions are connected, and the population can migrate freely between two regions. The number of population in region $\Omega_{1}$ is $x_{1}$, and $x_{2}$ is the number of population in region $\Omega_{2}$. The ultimate aim in harvesting the biological resources is to get economic profits and practical value. Generally, from the economic aspect, we know that the harvesting behavior changes with many market factors. Therefore, studying the relation between economic profits and the harvesting can help us better protect sustainable resources. If considering the economic profits in the model (2.1), the following mathematical model of the exploited population with protective region, called a singular ecological-economic model, is proposed:

$$
\begin{gathered}
\frac{d x_{1}}{d t}=\gamma x_{1}\left(1-\frac{x_{1}}{K_{1}}\right)-\alpha\left(x_{1}-x_{2}\right)-E x_{1}, \\
\frac{d x_{2}}{d t}=\gamma x_{2}\left(1-\frac{x_{2}}{K_{2}}\right)+\alpha\left(x_{1}-x_{2}\right), \\
0=\left(p x_{1}-c\right) E-m,
\end{gathered}
$$


where $p$ is the unit price, $c$ is the unit cost, $m$ is the economic profit, and $\alpha>0$ is the migration proportional coefficient between two regions. Considering the practical significance, $p$ and $c$ are positive constants. $K_{1}$ and $K_{2}$ are the environment carrying capacity of $\Omega_{1}$ and $\Omega_{2}$, respectively. The differential equations are the growth rate of the population in region $\Omega_{1}$ and $\Omega_{2}$. The algebraic equation is an economic model, which represents the relations of the total income, the total cost, and the economic profits.

Remark 2.1. In the management of sustainable resources, the model (2.2) not only considers the ecological balance but also includes the economic profits when the population is harvested, which combines the biological control problems with the economic problems. The model (2.2) provides an effective way for human being to maintain the ecological resources sustainable when we get economic profit.

Considering the biological significance, the model (2.2) is discussed in the following interval:

$$
R_{+}^{3}=\left\{X=\left(x_{1}, x_{2}, E\right) \mid x_{1} \geq 0, x_{2} \geq 0, E \geq 0\right\}
$$

If $m=0$, the economic equilibrium occur, that is, the income is equal to the cost. When the economic profit is zero, the population reaches the maximum harvesting effort, and it is called the overfishing. In the exploitation of population resources, a collapse of the population may occur.

Due to the limitation of the environment, the number of populations cannot exceed the environment maximum carrying capacity. Otherwise, due to the crowded environment, a large number of populations will die gradually. So the state variables and the parameters satisfy the following conditions:

$$
0<x_{1}<K_{1 \max }, \quad 0<x_{2}<K_{2 \max }, \quad 0<K_{1}<K_{1 \max }, \quad 0<K_{2}<K_{2 \max }, \quad 0<E<\gamma,
$$

where $K_{1 \max }$ and $K_{2 \max }$ are the maximum environment carrying capacities of $\Omega_{1}$ and $\Omega_{2}$, respectively.

\section{Local Stability Analysis}

For convenience, the environment carrying capacity in $\Omega_{1}$ is assumed to be proportional to that in $\Omega_{2}$, and the ratio is $\eta$, that is, $\eta K_{1}=K_{2}(\eta>0)$. For the model (2.2), the equilibrium points are the solutions for the equations:

$$
\begin{gathered}
r x_{1}\left(1-\frac{x_{1}}{K_{1}}\right)-\alpha\left(x_{1}-x_{2}\right)-E x_{1}=0 \\
r x_{2}\left(1-\frac{x_{2}}{\eta K_{1}}\right)+\alpha\left(x_{1}-x_{2}\right)=0 \\
\left(p x_{1}-c\right) E-m=0 .
\end{gathered}
$$


By solving (3.1), we get two equilibrium points for the model (2.2):

$$
\begin{gathered}
p_{0}=\left(0,0,-\frac{m}{c}\right), \\
p_{1}=\left(x_{10}, x_{20}, E_{0}\right)=\left(x_{0},\left(\frac{m}{\alpha\left(p x_{0}-c\right)}+1-\frac{\gamma}{\alpha}+\frac{\gamma x_{0}}{\alpha K_{1}}\right) x_{0}, \frac{m}{p x_{0}-c}\right) .
\end{gathered}
$$

Here $x_{0} \neq 0$ is the root of the equation:

$$
C_{0} x^{3}+C_{1} x^{2}+C_{2} x+C_{3}=0
$$

where $C_{0}=\gamma p\left(K_{1} \alpha-K_{1} \gamma+\gamma\right)^{2}, C_{1}=2 \eta K_{1} p m \gamma\left(K_{1} \alpha-K_{1} \gamma+\gamma\right)-2 p c \gamma\left(K_{1} \alpha-K_{1} \gamma+\gamma\right)^{2}, C_{2}=$ $\gamma c^{2}\left(K_{1} \alpha-K_{1} \gamma+\gamma\right)^{2}-2 \eta K_{1} c m \gamma\left(K_{1} \alpha-K_{1} \gamma+\gamma\right)+\alpha p \eta^{3} K_{1}^{3} m(\alpha-\gamma)+K_{1}^{2} m^{2} \gamma, C_{3}=-\alpha \eta^{3} K_{1}^{3} m c(\alpha-$ $\gamma)$.

When the coefficients $C_{i}(i=0,1,2,3)$ satisfy certain conditions, there is a positive solution for (3.3). Here, we suppose that the positive equilibrium point $p_{1}$ exists. We are interested in the local stability of the model (2.2) at the equilibrium points $p_{0}$ and the positive equilibrium point $p_{1}$. In order to analyze the local stability of the model (2.2), let

$$
\begin{gathered}
\mathbf{F}(\mathbf{X}, E)=\left(\begin{array}{c}
\gamma x_{1}\left(1-\frac{x_{1}}{K_{1}}\right)-\alpha\left(x_{1}-x_{2}\right)-E x_{1} \\
\gamma x_{2}\left(1-\frac{x_{2}}{\eta K_{1}}\right)+\alpha\left(x_{1}-x_{2}\right)
\end{array}\right) \\
G(\mathbf{X}, E)=\left(p x_{1}-c\right) E-m,
\end{gathered}
$$

where $\mathbf{X}=\left[\begin{array}{ll}x_{1} & x_{2}\end{array}\right]$. The local stability of the model (2.2) at the equilibrium points $p_{0}$ is discussed by the following theorem.

Theorem 3.1. If $0<\gamma<\alpha+m / 2 c$ and $\gamma(\gamma-2 \alpha)+(\gamma-\alpha+m / c)>0$, the model (2.2) is locally stable at $p_{0}$.

Proof. $p_{0}=(0,0,-m / c)$ is an equilibrium point of the model (2.2). Since $\left.\operatorname{det} D_{E} G\right|_{p_{0}}=-c \neq 0$, Jacobian matrix of the model (2.2) at $p_{0}$ is given by

$$
J=\left.\left[D_{\mathbf{X}} \mathbf{F}-D_{E} \mathbf{F}\left(D_{E} G\right)^{-1} D_{\mathbf{X}} G\right]\right|_{p_{0}}=\left[\begin{array}{cc}
\gamma-\alpha+\frac{m}{c} & \alpha \\
\alpha & \gamma-\alpha
\end{array}\right],
$$

where $D_{E} G$ denote the derivative of the function $G$ on the variable $E$.

The characteristic equation of Jacobian matrix (3.5) can be obtained:

$$
\lambda^{2}-\left[2(\gamma-\alpha)+\frac{m}{c}\right] \lambda+\gamma(\gamma-2 \alpha)+\left(\gamma-\alpha+\frac{m}{c}\right)=0 .
$$


If $0<\gamma<\alpha+m / 2 c$ and $\gamma(\gamma-2 \alpha)+(\gamma-\alpha+m / c)>0$, the roots of the characteristic equation (3.6) all have negative real part. Therefore, the model (2.2) is locally stable at $p_{0}$.

In order to analyze the local stability at the positive equilibrium point $p_{1}$, a linear transformation $\boldsymbol{X}^{T}=\mathbf{Q S}^{T}$ is used, where

$$
x=\left[\begin{array}{lll}
x_{1} & x_{2} & E
\end{array}\right], \quad \mathbf{S}=\left[\begin{array}{lll}
u & v & \bar{E}
\end{array}\right], \quad \mathbf{Q}=\left(\begin{array}{ccc}
1 & 0 & 0 \\
0 & 1 & 0 \\
-\frac{p E_{0}}{p x_{0}-c} & 0 & 1
\end{array}\right)
$$

Thus, $D_{X} G\left(x_{0}\right) \mathbf{Q}=\left[\begin{array}{lll}0 & 0 & p x_{0}-c\end{array}\right], u=x_{1}, v=x_{2}, \bar{E}=p E_{0} /\left(p x_{0}-c\right)+E$. The model (2.2) is changed into the following form:

$$
\begin{gathered}
\frac{d u}{d t}=\gamma u\left(1-\frac{u}{K_{1}}\right)-\alpha(u-v)-\bar{E} u+\frac{p E_{0}}{p x_{0}-c} u^{2}, \\
\frac{d v}{d t}=\gamma v\left(1-\frac{v}{\eta K_{1}}\right)+\alpha(u-v) \\
0=(p u-c)\left(\bar{E}-\frac{p E_{0}}{p x_{0}-c} u\right)-m .
\end{gathered}
$$

Now the local stability of the model (3.8) at the positive equilibrium point $p_{1}$ will be analyzed. First, the diffeomorphism $\psi$ is defined as follows:

$$
\left[\begin{array}{lll}
u & v & \bar{E}
\end{array}\right]^{T}=\psi(\overline{\mathbf{Z}})=\mathbf{S}_{0}^{T}+\mathbf{U}_{0} \overline{\mathbf{Z}}+\mathbf{V}_{0} h(\overline{\mathbf{Z}})
$$

where $\mathbf{U}_{0}=\left[\begin{array}{ll}1 & 0 \\ 0 & 1 \\ 0 & 0\end{array}\right], \mathbf{V}_{0}=\left[\begin{array}{l}0 \\ 0 \\ 1\end{array}\right], \overline{\mathbf{Z}}=\left[\begin{array}{ll}y_{1} & y_{2}\end{array}\right]^{T}, \mathbf{S}_{0}=\left[\begin{array}{ll}u_{0} & v_{0} \\ E_{0}\end{array}\right], h: R^{2} \rightarrow R^{1}$ is a smooth mapping. Jacobi matrix $D \psi$ is a $3 \times 1$ real matrix.

Second, by differentiating $G(\psi(\overline{\mathbf{Z}}))=0$, the following equation is obtained:

$$
D G(\chi) D \psi(\overline{\mathbf{Z}})=0
$$

Differentiating (3.9) and multiplying on the left by $\mathbf{U}_{0}^{T}$, it can be obtained that:

$$
\mathbf{U}_{0}^{T} D \psi(\overline{\mathbf{Z}})=\mathbf{I}_{2}
$$

where $\mathbf{I}_{2}$ is a $2 \times 2$ unit matrix. From (3.10) and (3.11), the following formula is gotten:

$$
D \psi(\overline{\mathbf{Z}})=\left[\begin{array}{c}
D G\left(\mathbf{S}_{0}\right) \\
\mathbf{U}_{0}^{T}
\end{array}\right]^{-1}\left[\begin{array}{l}
\mathbf{0} \\
\mathbf{I}_{2}
\end{array}\right]
$$


Furthermore, the following model is further obtained [37]:

$$
\frac{d \overline{\mathbf{Z}}}{d t}=\mathbf{U}_{0}^{T} f(\psi(\overline{\mathbf{Z}}))=\mathbf{U}_{0}^{T} D f\left(\boldsymbol{x}_{0}\right)\left[\begin{array}{c}
D G\left(\mathbf{S}_{0}\right) \\
\mathbf{U}_{0}^{T}
\end{array}\right]^{-1}\left[\begin{array}{l}
0 \\
\mathbf{I}_{2}
\end{array}\right]+Y(\overline{\mathbf{Z}})
$$

where $Y(\overline{\mathbf{Z}})=o(\overline{\mathbf{Z}})\left(\overline{\mathbf{Z}} \rightarrow 0^{+}\right)$.

From the transformation above and (3.13), the coefficient matrix of linear model corresponding to the model (3.8) is gotten as follows:

$$
\begin{aligned}
E\left(\mathbf{S}_{0}\right) & =\left(\begin{array}{c}
D_{S} f_{1}\left(\mathbf{S}_{0}\right) \\
D_{S} f_{2}\left(\mathbf{S}_{0}\right)
\end{array}\right)\left(\begin{array}{c}
D_{\mathbf{s}} G\left(\mathbf{S}_{0}\right) \\
\mathbf{U}_{0}^{T}
\end{array}\right)^{-1}\left(\begin{array}{ll}
0 & 0 \\
1 & 0 \\
0 & 1
\end{array}\right) \\
& =\left(\begin{array}{cc}
\gamma-\bar{E}_{0}-\alpha-\frac{2 \gamma u_{0}}{K_{1}}+\frac{2 p E_{0} u_{0}}{p x_{0}-c} & \alpha \\
\alpha & \gamma-\alpha-\frac{2 \gamma v_{0}}{\eta K_{1}}
\end{array}\right),
\end{aligned}
$$

where $u_{0}=x_{0}, v_{0}=\left(m / \alpha\left(p x_{0}-c\right)+1-\gamma / \alpha+\gamma x_{0} / \alpha K_{1}\right) x_{0}, \bar{E}_{0}=p E_{0} /\left(p x_{0}-c\right)+E_{0}$.

Thus, the characteristic equation of the matrix (3.14) is given by

$$
\lambda^{2}+D_{1} \curlywedge+D_{2}=0
$$

where $D_{1}=2(\alpha-\gamma)+\left(3 p E_{0} x_{0}+E_{0}(p-c)\right) /\left(p x_{0}-c\right)+2 \gamma x_{0} / \eta K_{1}\left(\left(m / \alpha\left(p x_{0}-c\right)\right)+2-(\gamma / \alpha)+\right.$ $\left.\left(\gamma / \alpha K_{1}\right)\right), D_{2}=\left[\gamma-\alpha+\left(3 p E_{0} x_{0}+E_{0}(p-c)\right) /\left(p x_{0}-c\right)\right]\left[\gamma-\alpha-\left(2 \gamma x_{0} / \eta K_{1}\right)\left(\left(m / \alpha\left(p x_{0}-c\right)\right)+\right.\right.$ $\left.\left.2-(\gamma / \alpha)+\left(\gamma / \alpha K_{1}\right)\right)\right]$.

About the local stability of the model (2.2) at the positive equilibrium point $p_{1}$, we have the following theorem.

Theorem 3.2. For the model (2.2):

(a) if $D_{1}>0$ and $D_{2}>0$, the model (2.2) is locally stable at the positive equilibrium point $p_{1}$;

(b) if $D_{1}<0$ or $D_{2}<0$, the model (2.2) is unstable at the positive equilibrium point $p_{1}$.

Proof. The model (3.8) and the model (2.2) are isomorphic. The local stability of them is discussed by the eigenvalues of the coefficient matrix $E\left(\mathbf{S}_{0}\right)$. When $D_{1}>0$ and $D_{2}>0$, two roots of the characteristic equation (3.15) all have negative real part. The model (3.8) and the model (2.2) are all locally stable at the positive equilibrium point $p_{1}$.

However, when $D_{1}<0$ or $D_{2}<0$, at least one of the eigenvalues of $E\left(\mathbf{S}_{0}\right)$ has nonnegative real part. We can conclude that the model (2.2) is unstable at the positive equilibrium point $p_{1}$. Thus, the proof is completed. 
To further study the dynamic behavior of the model (2.2), $x_{0}$ is given a specified value. If $x_{0}=c / p$, the positive equilibrium point of the model (2.2) is

$$
p_{1}\left(x_{10}, x_{20}, E_{0}\right)=\left(\frac{c}{p^{\prime}} \frac{\gamma-\alpha+\theta}{2 p \gamma},(\gamma-\alpha)\left(\frac{\alpha}{2 c \gamma}+1\right)-\frac{\gamma c}{p K_{1}}+\frac{\alpha \theta}{2 c \gamma}\right)
$$

where $\theta=\sqrt{(\gamma-\alpha)^{2} p^{2} \eta^{2} K_{1}^{2}+4 p \eta K_{1} \gamma \alpha c}$. By analysis, we know that there is a bifurcation at the positive equilibrium point $p_{1}$ for the model (2.2), which is shown in the following theorem.

Theorem 3.3. If $\gamma-(\gamma-\alpha+\theta) / K_{1} p-\alpha \neq 0$, there is a singular induced bifurcation for the model (2.2) at the positive equilibrium point $p_{1}$, and $m=0$ is a bifurcation value.

Proof. Let $m$ be a bifurcation parameter for the model (2.2). $x_{1}=c / p$ makes $\Delta=\operatorname{det}\left[D_{E} G\right]=$ $p x_{1}-c=0$. If $\gamma-(\gamma-\alpha+\theta) / K_{1} p-\alpha \neq 0$, the following three conditions are satisfied:

(i) $\operatorname{trace}\left[D_{E} F \operatorname{adj}\left(D_{E} G\right)\left(D_{x_{1}} G \quad D_{x_{2}} G\right)\right]_{p_{1}}$

$$
\begin{aligned}
& =\left(\begin{array}{cc}
-p E x_{1} & 0 \\
0 & 0
\end{array}\right)_{p_{1}} \\
& =-c(\gamma-\alpha)\left(\frac{\alpha}{2 c \gamma}+1\right)+\frac{\gamma c^{2}}{p K_{1}}-\frac{\alpha \theta}{2 \gamma} \neq 0 ;
\end{aligned}
$$

(ii) $\left|\begin{array}{cc}D_{\mathbf{X}} \mathbf{F} & D_{E} \mathbf{F} \\ D_{\mathbf{X}} G & D_{E} G\end{array}\right|_{p_{0}}$

$$
\begin{aligned}
& =\left|\begin{array}{ccc}
\gamma-\frac{2 \gamma}{K_{1}} x_{1}-\alpha-E & \alpha & -x_{1} \\
\alpha & \gamma-\frac{2 \gamma}{\eta K_{1}} x_{2}-\alpha & 0 \\
p E & 0 & p x_{1}-c
\end{array}\right|_{p_{1}} \\
& =\left[c(\gamma-\alpha)\left(\frac{\alpha}{2 c \gamma}+1\right)-\frac{\gamma c^{2}}{p \eta K_{1}}+\frac{\alpha \theta}{2 \gamma}\right]\left(\gamma-\frac{\gamma-\alpha+\theta}{K_{1} p}-\alpha\right) \neq 0 ;
\end{aligned}
$$

(iii) $\left|\begin{array}{lll}D_{\mathbf{X}} \mathbf{F} & D_{E} \mathbf{F} & D_{m} \mathbf{F} \\ D_{\mathbf{X}} G & D_{E} G & D_{m} G \\ D_{\mathbf{X}} \Delta & D_{E} \Delta & D_{m} \Delta\end{array}\right|_{p_{0}}$

$$
\begin{aligned}
& =\left|\begin{array}{cccc}
\gamma-\frac{2 \gamma}{K_{1}} x_{1}-\alpha-E & \alpha & -x_{1} & 0 \\
\alpha & \gamma-\frac{2 \gamma}{\eta K_{1}} x_{2}-\alpha & 0 & 0 \\
p E & 0 & p x_{1}-c & -1 \\
p & 0 & 0 & 0
\end{array}\right|_{p_{1}} \\
& =-\frac{c}{p}\left(\gamma-\frac{\gamma-\alpha+\theta}{K_{1} p}-\alpha\right) \neq 0 .
\end{aligned}
$$


Thus, we can conclude that there exists a smooth curve in $\mathbf{R}^{3}$ which passes through the positive equilibrium point $p_{1}$, and it is transversal to the singular surface at the positive equilibrium point $p_{1}$. And we can get the following equations:

$$
\begin{aligned}
& i=-\operatorname{trace}\left[D_{E} F \operatorname{adj}\left(D_{E} G\right)\left(D_{x_{1}} G D_{x_{2}} G\right)\right]_{p_{1}}=c(\gamma-\alpha)\left(\frac{\alpha}{2 c \gamma}+1\right)-\frac{\gamma c^{2}}{p K_{1}}+\frac{\alpha \theta}{2 \gamma} ; \\
& j=D_{m} \Delta-\left(\begin{array}{ll}
D_{\mathbf{X}} \Delta & D_{E} \Delta
\end{array}\right)\left(\begin{array}{cc}
D_{\mathbf{X}} F & D_{E} F \\
D_{\mathbf{X}} G & D_{E} G
\end{array}\right)^{-1}\left(\begin{array}{c}
D_{m} F \\
D_{m} G
\end{array}\right) \\
& =-\left(\begin{array}{lll}
p & 0 & 0
\end{array}\right)\left(\begin{array}{ccc}
\gamma-\frac{2 \gamma}{K_{1}} x_{1}-\alpha-E & \alpha & -x_{1} \\
\alpha & \gamma-\frac{2 \gamma}{\eta K_{1}} x_{2}-\alpha & 0 \\
p E & 0 & p x_{1}-c
\end{array}\right)_{p_{1}}^{-1}\left(\begin{array}{c}
0 \\
0 \\
-1
\end{array}\right) \\
& =\frac{1}{(\gamma-\alpha)(\alpha / 2 c \gamma+1)-\gamma c / p K_{1}+\alpha \theta / 2 c \gamma} \text {. }
\end{aligned}
$$

From above we can get that $i / j=c\left[(\gamma-\alpha)(\alpha / 2 c \gamma+1)-\gamma c / p K_{1}+\alpha \theta / 2 c \gamma\right]^{2}$. Obviously, $i / j>0$. According to Theorem 3 in [38], when $m$ passes through 0 , one eigenvalue of matrix $J=D_{\mathrm{X}} F-D_{E} F\left(D_{E} G\right)^{-1} D_{\mathrm{X}} G$ moves from $C^{-}$to $C^{+}$along the real axis by diverging through $\infty$. There is a singular induced bifurcation for the model (2.2), and the model turns to unstable. The proof is completed.

Remark 3.4. When the economic profit is zero, it is called the overfishing in economics. One eigenvalue of the model (2.2) is approaching to endless, and the impulse occurs in the model (2.2). This would lead to the collapse of the population and destroy the ecological balance. It is necessary to find an effective method to make that the population develop sustainably.

\section{Controller Design}

Variable structure control is often used to deal with some models with internal varying parameters and external disturbances since it provides effective means to design robust state feedback controllers. In this section, variable structure control is introduced to eliminate the bifurcation behavior and ensure the system stable. This approach makes direct use of the nonlinear model and the full biological state information. In order to facilitate the controller design, differentiating the second differential equation in the model (2.2) and substituting the other two equations into it, the model (2.2) is transformed into a second-order differential equation [39]:

$$
\begin{aligned}
\frac{d^{2} x_{2}}{d t^{2}} & +\left(\alpha+\frac{2 \gamma x_{2}}{K_{2}}-\gamma\right) \frac{d x_{2}}{d t}-\alpha^{2} x_{2} \\
& =\frac{K_{1} p E\left(\alpha \gamma m-E c \alpha \gamma-\alpha^{2} m-\alpha^{2} c E-\alpha m E\right)-\alpha \gamma m(m+c E+c E)-c \alpha^{2} \gamma E^{2}}{K_{1} p^{2} E^{2}}+\frac{c}{p} E
\end{aligned}
$$


Equation (4.1) can be rewritten as a single-input and single-output model with the parameters varying within definite intervals:

$$
\frac{d^{2} y}{d t^{2}}+a_{1} \frac{d y}{d t}+a_{0} y=b_{0} u+\beta
$$

where $y=x_{2}, u=E, a_{1}=\alpha+2 \gamma x_{2} / K_{2}-\gamma, a_{0}=-\alpha^{2}, b_{0}=c / p, \beta=\left(K_{1} p E\left(\alpha \gamma m-E c \alpha \gamma-\alpha^{2} m-\right.\right.$ $\left.\left.\alpha^{2} c E-\alpha m E\right)-\alpha \gamma m(m+2 c E)-c \alpha^{2} \gamma E^{2}\right) / K_{1} p^{2} E^{2}$.

Obviously, $a_{0}$ and $b_{0}$ are fixed, while $a_{1}$ and $\beta$ change with the parameters and the variables. From the varying intervals (2.4), we can get the varying intervals of the coefficients $a_{1}$ and $\beta$ :

$$
\begin{gathered}
\alpha-\gamma<a_{1}<\alpha+\gamma \\
\frac{-c \alpha \gamma-\left(\alpha^{2}+\alpha\right) m}{p}<\beta<\frac{\left(\alpha \gamma-\alpha^{2}\right) m}{p \gamma}-\frac{\left(\alpha \gamma+\alpha^{2}\right) c+\alpha m}{p}-\frac{\alpha m(m+2 c \gamma)}{K_{1} p^{2} \gamma}-\frac{c \alpha^{2} \gamma}{K_{1} p^{2}}
\end{gathered}
$$

In order to make the number of the population in protecting region $\Omega_{2}$ reach the carrying capacity, let

$$
e=K_{2}-y
$$

where $e$ is the error of $y$ and $K_{2}$. Here $y$ is the number of population in $\Omega_{2}$, while $K_{2}$ is the carrying capacity of region $\Omega_{2}$.

Differentiating the formula (4.4) twice and considering the model (4.2), the following equation is obtained:

$$
\frac{d^{2} e}{d t^{2}}+a_{1} \frac{d e}{d t}+a_{0} e=-b_{0} u+\left(a_{0} K_{2}-\beta\right)
$$

For the differential equation (4.5), $a_{0} K_{2}-\beta$ is considered as an external disturbance. According to the transformation, the model (4.1) is considered as a linear uncertain system with the control input. And then the model (4.5) is transformed into

$$
\begin{aligned}
& \frac{d e_{1}}{d t}=e_{2}, \\
& \frac{d e_{2}}{d t}=-a_{0} e_{1}-a_{1} e_{2}-b_{0} u+a_{0} K_{2}-\beta
\end{aligned}
$$

where $e_{1}=e$.

The model (4.6) can be rewritten as a matrix form:

$$
\frac{d \mathbf{w}}{d t}=\mathbf{A} \mathbf{w}+\mathbf{B} u+\mathbf{C K}_{2}+\mathbf{D},
$$

where $\mathbf{w}=\left[\begin{array}{ll}e_{1} & e_{2}\end{array}\right]^{T}, \mathbf{A}=\left(\begin{array}{cc}0 & 1 \\ -a_{0} & -a_{1}\end{array}\right), \mathbf{B}=\left[\begin{array}{ll}0 & -b_{0}\end{array}\right]^{T}, \mathbf{C}=\left[\begin{array}{ll}0 & a_{0}\end{array}\right]^{T}$, and $\mathbf{D}=\left[\begin{array}{ll}0 & -\beta\end{array}\right]^{T}$. 
To stabilize the model (4.7), the variable structure controller is designed as

$$
u=-\left(\lambda_{1} e_{1}+\lambda_{2} e_{2}+\lambda_{3} K_{2}\right) \operatorname{Sgn}(\delta(\mathbf{w}))
$$

where $\lambda_{i}(i=1,2,3)$ are switching coefficients and $\operatorname{Sgn}(\delta(\mathbf{w}))$ is a sign function. $\delta(\mathbf{w})$ is called sliding surface, which divides the phase plane into two regions. The function $\delta(\mathbf{w})$ contains only endpoints of the trajectories of the model (4.7) coming from both sides of the surface and is defined as

$$
\delta(\mathbf{w})=f e_{1}+e_{2}
$$

where $f>0$ is a constant. To suppress the effect of the uncertainty and drive the trajectories of the model (4.7) toward the sliding surface until intersection occurs, the following reachable condition is established:

$$
\delta(\mathbf{w}) \frac{d \delta(\mathbf{w})}{d t}<0, \quad \text { for } \delta(\mathbf{w}) \neq 0
$$

That is

$$
\frac{d \delta(\mathbf{w})}{d t}=f \frac{d e_{1}}{d t}+\frac{d e_{2}}{d t}= \begin{cases}\left(\lambda_{1}-a_{0}\right) e_{1}+\left(f+\lambda_{2}-a_{1}\right) e_{2}+\left(a_{0}+\lambda_{3}\right) K_{2}<0, & \delta(\mathbf{w})>0 \\ \left(-\lambda_{1}-a_{0}\right) e_{1}+\left(f+\lambda_{2}-a_{1}\right) e_{2}+\left(a_{0}-\lambda_{3}\right) K_{2}>0, & \delta(\mathbf{w})<0 .\end{cases}
$$

According to the reachable condition (4.10), we get the variable structure controller for the model (4.7):

$$
u= \begin{cases}u^{+}=-\left(\lambda_{1} e_{1}+\lambda_{2} e_{2}+\lambda_{3} K_{2}\right), & \delta(\mathbf{w})>0 \\ u^{-}=\lambda_{1} e_{1}+\lambda_{2} e_{2}+\lambda_{3} K_{2}, & \delta(\mathbf{w})<0\end{cases}
$$

Using the controller $u=u^{+}$in the model (4.7), the controlled model is

$$
\left[\begin{array}{l}
\frac{d e_{1}}{d t} \\
\frac{d e_{2}}{d t}
\end{array}\right]=\left[\begin{array}{cc}
0 & 1 \\
\lambda_{1}-a_{0} & \lambda_{2}-a_{1}
\end{array}\right]\left[\begin{array}{l}
e_{1} \\
e_{2}
\end{array}\right]+\left[\begin{array}{c}
0 \\
a_{0}+\lambda_{3}
\end{array}\right] K_{2}+\left[\begin{array}{c}
0 \\
-\beta
\end{array}\right] .
$$

Let $e_{1}^{\prime}=e_{1}+\left(\lambda_{3}+a_{0}\right) K_{2} /\left(\lambda_{1}-a_{0}\right)$ and $e_{2}^{\prime}=e_{2}$, then

$$
\left[\begin{array}{c}
\frac{d e_{1}^{\prime}}{d t} \\
\frac{d e_{2}^{\prime}}{d t}
\end{array}\right]=\left[\begin{array}{cc}
0 & 1 \\
\lambda_{1}-a_{0} & \lambda_{2}-a_{1}
\end{array}\right]\left[\begin{array}{c}
e_{1}^{\prime} \\
e_{2}^{\prime}
\end{array}\right]+\left[\begin{array}{c}
0 \\
-\beta
\end{array}\right]
$$


Obviously, the model (4.13) and the model (4.14) have the same state matrix $M=$ $\left[\begin{array}{cc}0 & 1 \\ \lambda_{1}-a_{0} & \lambda_{2}-a_{1}\end{array}\right] . \beta$ is a bounded constant, and it does not influence the local stability of the controlled model. Thus, we have the following theorem.

Theorem 4.1. If $a_{0}-\lambda_{1}>0, a_{1}-\lambda_{2}>0$, the model (4.7) can be stabilized by the controller $u^{+}$.

Proof. When the model (4.7) is controlled by the controller $u^{+}$, it is transformed into the linear model (4.13). The characteristic equation of the state matrix $M$ is

$$
|\lambda E-M|=\lambda^{2}-\left(\lambda_{2}-a_{1}\right) \lambda-\left(\lambda_{1}-a_{0}\right)=0 .
$$

According to the Routh-Hurwitz criterion, if $a_{0}-\lambda_{1}>0, a_{1}-\lambda_{2}>0$, two eigenvalues for the state matrix $M$ have negative real part. Therefore, the model (4.13) is locally stable. That is to say, the model (4.7) can be stabilized by the controller $u^{+}$.

If $u=u^{-}$in the model (4.7), the controlled model is

$$
\left[\begin{array}{l}
\frac{d e_{1}}{d t} \\
\frac{d e_{2}}{d t}
\end{array}\right]=\left[\begin{array}{cc}
0 & 1 \\
-\lambda_{1}-a_{0} & -\lambda_{2}-a_{1}
\end{array}\right]\left[\begin{array}{l}
e_{1} \\
e_{2}
\end{array}\right]+\left[\begin{array}{c}
0 \\
a_{0}-\lambda_{3}
\end{array}\right] K_{2}+\left[\begin{array}{c}
0 \\
-\beta
\end{array}\right]
$$

Let $e_{1}^{\prime}=e_{1}+\left(\lambda_{3}-a_{0}\right) K_{2} /\left(\lambda_{1}+a_{0}\right)$ and $e_{2}^{\prime}=e_{2}$, then,

$$
\left[\begin{array}{l}
\frac{d e_{1}^{\prime \prime}}{d t} \\
\frac{d e_{2}^{\prime \prime}}{d t}
\end{array}\right]=\left[\begin{array}{cc}
0 & 1 \\
-\lambda_{1}-a_{0} & -\lambda_{2}-a_{1}
\end{array}\right]\left[\begin{array}{c}
e_{1}^{\prime \prime} \\
e_{2}^{\prime \prime}
\end{array}\right]+\left[\begin{array}{c}
0 \\
-\beta
\end{array}\right]
$$

The model (4.16) and the model (4.17) also have the same state matrix $N=$ $\left[\begin{array}{cc}0 & 1 \\ -\lambda_{1}-a_{0} & -\lambda_{2}-a_{1}\end{array}\right]$. The transformation does not change the local stability of the model (4.16). Furthermore, we have another theorem.

Theorem 4.2. If $\lambda_{1}+a_{0}>0, \lambda_{2}+a_{1}>0$, the model (4.7) can be stabilized by the controller $u^{-}$.

Proof. When the model (4.7) is controlled by the controller $u^{-}$, it is transformed into the linear model (4.16). The characteristic equation of the state matrix $N$ is

$$
|\lambda E-N|=\lambda^{2}-\left(\lambda_{2}+a_{1}\right) \lambda-\left(\lambda_{1}+a_{0}\right)=0 .
$$

According to the Routh-Hurwitz criterion, if $\lambda_{1}+a_{0}>0, \lambda_{2}+a_{1}>0$, two eigenvalues for the state matrix $N$ have negative real part. The model (4.16) is locally stable, and the model (4.7) can be stabilized by the controller $u^{-}$. 
From the condition (4.11), Theorems 4.1 and 4.2, we get the varying range of the switching coefficients $\lambda_{i}(i=1,2,3)$ :

$$
\lambda_{1} \geq \max \left|-a_{0}\right|, \quad \lambda_{2} \geq \max \left|f-a_{1}\right|, \quad \lambda_{3} \geq \max \left|a_{0}\right| .
$$

According to the condition $\delta(\mathbf{w})=0$ and $d \delta(\mathbf{w}) / d t=0$, the equivalent control on the sliding surface $\delta(\mathbf{w})=0$ can be obtained. If $\delta(\mathbf{w})=0$, there is a state variable represented by the remaining state variables. From the condition $\operatorname{Sgn}(\delta(\mathbf{w}))=0$, we have

$$
\frac{d e_{1}}{d t}=-f e_{1}
$$

Remark 4.3. When applying variable structure control, the singular model is transformed into a linear model with parameters varying within definite intervals. Since the sliding surface can be designed as required and has nothing to do with the parameters and disturbance, it makes the discontinuous control insensitive to internal parameter variations and extraneous disturbance and decreases the chattering phenomenon. Variable structure control can stabilize the nonlinear system effectively.

\section{Simulations}

Fishery production is an important aspect in human life. In order to guarantee the sustainable development of the fishery, people have taken many necessary measures. Therefore, to study the structure model for the inshore-offshore fishery is necessary. It is a good idea to divide the population into two categories in keeping resources sustainable, a harvesting-permitted category and a harvesting-forbidden category. Some inshore-offshore models in an aquatic environment have ever been studied to keep the fishery sustainable [40-42]. But these papers did not consider the economic profits that the fishery brings for people. In this paper, the sustainable fishery and the economic interest are discussed for the inshore-offshore fishery model.

The sea around Zhoushan is a famous fishing ground in Zhejiang province. The total sea area is about more than $10800 \mathrm{~km}^{2}$. The area of the inshore region is about $3700 \mathrm{~km}^{2}$, and the offshore region is about $7100 \mathrm{~km}^{2}$ [43]. The coiliaspp is a kind of fish, and it is about 1099 million in the whole sea area [44]. To protect the fishery resources, the coiliaspp in the inshore region is permitted to be harvested, while the offshore region is forbidden. In the inshore region, the density of the coiliaspp is greater than that in the offshore region because of the environment effect. So the environment carrying capacity of the inshore region is about 423 million, and the offshore environment carrying capacity is 676 million. The intrinsic growth rate $\gamma$ is assumed to be 0.2 . When the number of the fish in two regions are different, they migrate between two regions at the proportional $\alpha=0.6$. It is supposed that they are sold at the average unit price $p=11$, and its unit $\operatorname{cost} c$ is 6 . 
Considering these conditions, the following singular ecological-economic model can be established:

$$
\begin{gathered}
\frac{d x_{1}}{d t}=0.2 x_{1}\left(1-\frac{x_{1}}{423}\right)-0.6\left(x_{1}-x_{2}\right)-E x_{1}, \\
\frac{d x_{2}}{d t}=0.2 x_{2}\left(1-\frac{x_{2}}{676}\right)+0.6\left(x_{1}-x_{2}\right), \\
0=\left(11 x_{1}-6\right) E-m .
\end{gathered}
$$

When the economic profit $m$ varies, there are some complex dynamic behaviors for the model (5.1), such as the singular induced bifurcation. When the economic profit $m=0$, the model $(5.1)$ has a positive equilibrium point $p^{*}(0.545,0.818,0.499)$. When economic profit $m=0.001$, there are two eigenvalues for the matrix $J=D_{\mathbf{X}} F-D_{E} F\left(D_{E} G\right)^{-1} D_{\mathbf{X}} G,-1.2998$ and -0.0002 . The eigenvalues became -1.2998 and 0.0017 when the parameter $m=-0.001$. It obvious that one eigenvalue remains constant, and the other eigenvalue moves from $C^{-}$ to $C^{+}$along the real axis by diverging through $\infty$. It is called the overexploitation, and it causes the extinction of the coiliaspp. In order to avoid such phenomena, a variable structure controller is designed to make the coiliaspp in the offshore region reach the environment carrying capacity 676 million. According to the varying range of the switching coefficients (4.19), the variable structure controller is designed as follows:

$$
u=-\left(10 e_{1}+76 e_{2}+15800\right) \operatorname{Sgn}(\delta(\mathbf{w})),
$$

where the sliding surface is chosen as $\delta(\mathbf{w})=9.6 e_{1}+e_{2}$. By controlling the harvesting effort $E, x_{2}$ reaches the environment carrying capacity 676 million in $\Omega_{2}$. Figure 2 shows the control result of $x_{1}, x_{2}$, and $E$ with variable structure control.

In Figure 2, when the harvesting effort $E$ is controlled at 0.16 million, the number of fish in region $\Omega_{2}$ reaches 675.2 million controlled by the controller $u$. Due to the migration between $\Omega_{1}$ and $\Omega_{2}$, the coiliaspp in inshore region reaches 424.3 million accordingly. The state variables stay in a stable situation, and the singular induced bifurcation is eliminated by the controller $u$. In practical, we can regulate the harvesting behavior by the revenue to keep the harvesting and the reproduction in balance. Therefore, the sustainable development of the fishery can be realized by this controller. Further, we know that the corresponding nonlinear singular ecological-economic model can be stabilized by variable structure control.

\section{Conclusions}

In this paper, the population is divided into the harvesting region and the protecting region, in which the population can migrate between two regions. In harvesting the population resources, when the economic interest and the environmental effects are taken into account, a singular ecological-economic model is established. The local stability and the dynamic behavior for this model are discussed. As the parameters changing, the singular model undergoes the singular induced bifurcation. In order to apply variable structure control to eliminate this complex behavior, the singular model is transformed into a linear singleinput and single-output model with parameters varying within definite intervals. Variable 


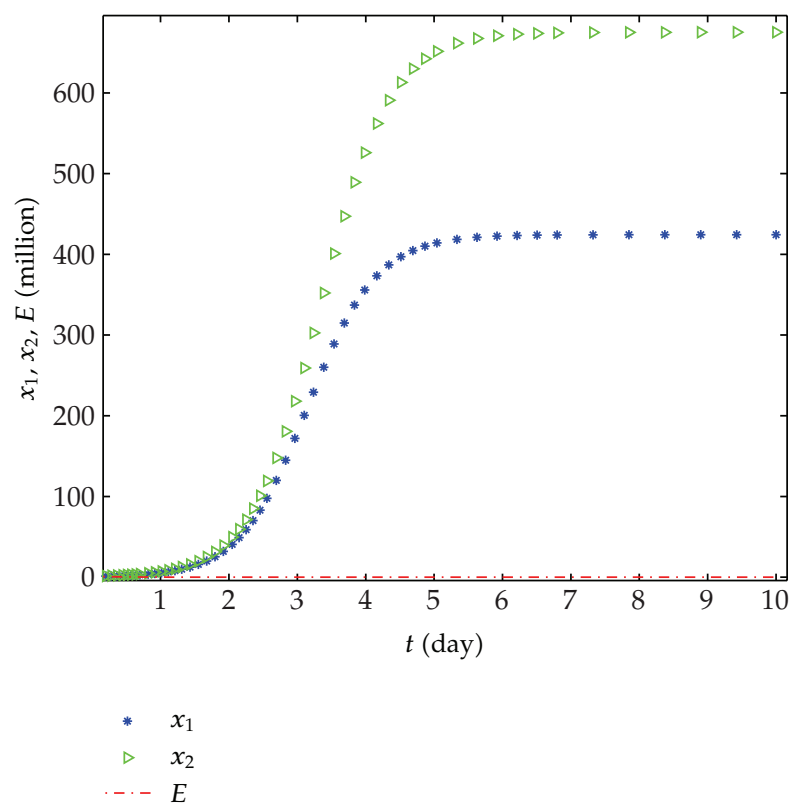

Figure 2: The state response of $x_{1}, x_{2}$, and $E$ when $m=1325$ with the controller $u$.

structure control with sliding mode is designed to stabilize the model. An inshore-offshore fishery model illustrates the analysis result. Some simulations show the effectiveness of the control method.

\section{Acknowledgments}

This work was supported by the National Natural Science Foundation of China (60974004).

\section{References}

[1] W. G. Marszalek and Z. W. Trzaska, "Singularity-induced bifurcations in electrical power systems," IEEE Transactions on Power Systems, vol. 20, no. 1, pp. 312-320, 2005.

[2] M. Yue and R. Schlueter, "Bifurcation subsystem and its application in power system analysis," IEEE Transactions on Power Systems, vol. 19, no. 4, pp. 1885-1893, 2004.

[3] T. Y. Guo and R. A. Schlueter, "Identification of generic bifurcation and stability problems in power system differential-algebraic model," IEEE Transactions on Power Systems, vol. 9, no. 2, pp. 1032-1038, 1994.

[4] R. Riaza and P. J. Zufiria, "Differential-algebraic equations and singular perturbation methods in recurrent neural learning," Dynamical Systems, vol. 18, no. 1, pp. 89-105, 2003.

[5] Z. W. Gao and S. X. Ding, "State and disturbance estimator for time-delay systems with application to fault estimation and signal compensation," IEEE Transactions on Signal Processing, vol. 55, no. 12, pp. 5541-5551, 2007.

[6] Z. Gao and D. W. C. Ho, "State/noise estimator for descriptor systems with application to sensor fault diagnosis," IEEE Transactions on Signal Processing, vol. 54, no. 4, pp. 1316-1326, 2006.

[7] R. J. Spiteri, U. M. Ascher, and D. K. Pai, "Numerical solution of differential systems with algebraic inequalities arising in robot programming," in Proceedings of the IEEE International Conference on Robotics and Automation, pp. 2373-2380, May 1995. 
[8] R. J. Spiteri, D. K. Pai, and U. M. Ascher, "Programming and control of robots by means of differential algebraic inequalities," IEEE Transactions on Robotics and Automation, vol. 16, no. 2, pp. 135-145, 2000.

[9] W. M. Liu, H. W. Hethcote, and S. A. Levin, "Dynamical behavior of epidemiological models with nonlinear incidence rates," Journal of Mathematical Biology, vol. 25, no. 4, pp. 359-380, 1987.

[10] N. Yi, Z. Zhao, and Q. Zhang, "Bifurcations of an SEIQS epidemic model," International Journal of Information E Systems Sciences, vol. 5, no. 3-4, pp. 296-310, 2009.

[11] N. Yi, Q. Zhang, K. Mao, D. Yang, and Q. Li, “Analysis and control of an SEIR epidemic system with nonlinear transmission rate," Mathematical and Computer Modelling, vol. 50, no. 9-10, pp. 1498-1513, 2009.

[12] D. G. Luenberger and A. Arbel, "Singular dynamic Leontief system," Econometrics, vol. 45, no. 32, pp. 991-995, 1997.

[13] J. S. Zhang, Singular System Economic Cybernetics, Tsinghua University Press, Beijing, China, 1990.

[14] A. Kumar and P. Daoutidis, Control of Nonlinear Differential Algebraic Equation Systems, vol. 397 of Chapman \& Hall/CRC Research Notes in Mathematics, Chapman \& Hall/CRC, Boca Raton, Fla, USA, 1999.

[15] Y. Zhang, Q. L. Zhang, and L. C. Zhao, "Bifurcations and control in singular biological economic model with stage structure," Journal of Systems Engineering, vol. 22, no. 3, pp. 233-238, 2007.

[16] C. Liu, Q. L. Zhang, Y. Zhang, and X. D. Duan, "Bifurcation and control in a differential-algebraic harvested prey-predator model with stage structure for predator," International Journal of Bifurcation and Chaos, vol. 18, no. 10, pp. 3159-3168, 2008.

[17] Y. Zhang and Q. Zhang, "Optimal control of logistic bioeconomic model with singularity-induced bifurcation," International Journal of Information E Systems Sciences, vol. 5, no. 3-4, pp. 369-379, 2009.

[18] C. Liu, Q. Zhang, and X. Zhang, "Dynamic analysis in a harvested differential-algebraic prey-predator model," Journal of Mechanics in Medicine and Biology, vol. 9, no. 1, pp. 123-140, 2009.

[19] X. Zhang, Q.-1. Zhang, and Y. Zhang, "Bifurcations of a class of singular biological economic models," Chaos, Solitons \& Fractals, vol. 40, no. 3, pp. 1309-1318, 2009.

[20] H. S. Gordon, "The economic theory of a common property resource: the Fishery," Journal of Political Economy, vol. 62, pp. 124-142, 1954.

[21] W. J. Reed, "Recruitment variability and age structure in harvested animal populations," Mathematical Biosciences, vol. 65, no. 2, pp. 239-268, 1983.

[22] T. K. Kar, "Modelling and analysis of a harvested prey-predator system incorporating a prey refuge," Journal of Computational and Applied Mathematics, vol. 185, no. 1, pp. 19-33, 2006.

[23] L. Liu and J. Sun, "Existence of periodic solution for a harvested system with impulses at variable times," Physics Letters, Section A, vol. 360, no. 1, pp. 105-108, 2006.

[24] M. Dieter, "Analysis of trade in illegally harvested timber: accounting for trade via third party countries," Forest Policy and Economics, vol. 11, no. 8, pp. 600-607, 2009.

[25] S. V. Emelyanov, Variable Structure Control Systems, Nauka, Moscow, Russia, 1967.

[26] Y. Itkis, Control Systems of Variable Structure, John Wiley \& Sons, New York, NY, USA, 1976.

[27] V. I. Utkin, Sliding Modes and Their Application in Variable Structure Systems, Nauka, Moscow, Russia, 1978.

[28] R. Suarez-Cortez, J. Alvarez-Gallegns, and E. Gonzalez-Mora, "Sliding controller design for a nonlinear fermentation system," Biotechnology and Bioengineering, vol. 33, no. 4, pp. 377-385, 1989.

[29] A. S. Zinober, Deterministic Control of Uncertain Systems, vol. 40 of IEE Control Engineering Series, Peter Peregrinus, London, UK, 1990.

[30] V. I. Utkin, Sliding Modes in Control and Optimization, Communications and Control Engineering Series, Springer, Berlin, Geramny, 1992.

[31] P. Park, D. J. Choi, and S. G. Kong, "Output feedback variable structure control for linear systems with uncertainties and disturbances," Automatica, vol. 43, no. 1, pp. 72-79, 2007.

[32] C.-C. Wang and J.-P. Su, "A new adaptive variable structure control for chaotic synchronization and secure communication," Chaos, Solitons \& Fractals, vol. 20, no. 5, pp. 967-977, 2004.

[33] F. Juan and L. Gerard, "Variable structure control for power systems stabilization," International Journal of Electrical Power and Energy Systems, vol. 32, no. 2, pp. 101-107, 2010.

[34] C.-C. Wang and J.-P. Su, "A new adaptive variable structure control for chaotic synchronization and secure communication," Chaos, Solitons \& Fractals, vol. 20, no. 5, pp. 967-977, 2004.

[35] S. H. Ling, F. H. F. Leung, H. K. Lam, and H. H. C. Iu, "Variable-structure neural network with realcoded genetic algorithm and its application on short-term load forecasting," International Journal of Information and Systems Sciences, vol. 5, no. 1, pp. 23-40, 2009. 
[36] J. Fernández-Vargas and G. Ledwich, "Variable structure control for power systems stabilization," International Journal of Electrical Power and Energy Systems, vol. 32, no. 2, pp. 101-107, 2010.

[37] B. S. Chen, X. X. Liao, and Y. Q. Liu, "Normal forms and bifurcations for differential-algebraic systems," Acta Mathematicae Applicatae Sinica, vol. 23, no. 3, pp. 429-443, 2000 (Chinese).

[38] V. Venkatasubramanian, H. Schättler, and J. Zaborszky, "Local bifurcations and feasibility regions in differential-algebraic systems," IEEE Transactions on Automatic Control, vol. 40, no. 12, pp. 1992-2013, 1995.

[39] P. Zlateva, "Variable-structure control of nonlinear systems," Control Engineering Practice, vol. 4, no. 7, pp. 1023-1028, 1996.

[40] T. Pradhan and K. S. Chaudhuri, "Bioeconomic modelling of selective harvesting in an inshore-offshore fishery," Differential Equations and Dynamical Systems, vol. 7, no. 3, pp. 305-320, 1999.

[41] B. Dubey, P. Chandra, and P. Sinha, "A model for fishery resource with reserve area," Nonlinear Analysis: Real World Applications, vol. 4, no. 4, pp. 625-637, 2003.

[42] M. Jerry, N. Raissi, and A. Rapaport, "A viability analysis for an explicit inshore-offshore model," Journal of Applied Mathematics, vol. 1, no. 1, pp. 41-60, 2011.

[43] http://wiki.cnki.com.cn/HotWord/7044734.htm.

[44] http://info.china.alibaba.com/news/detail/v0-d1023779518.html. 


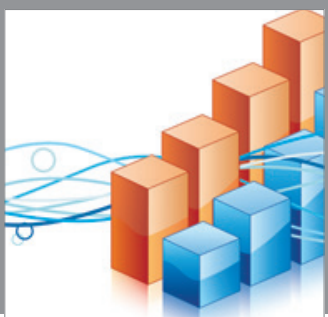

Advances in

Operations Research

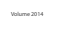

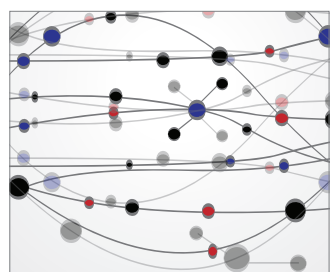

\section{The Scientific} World Journal
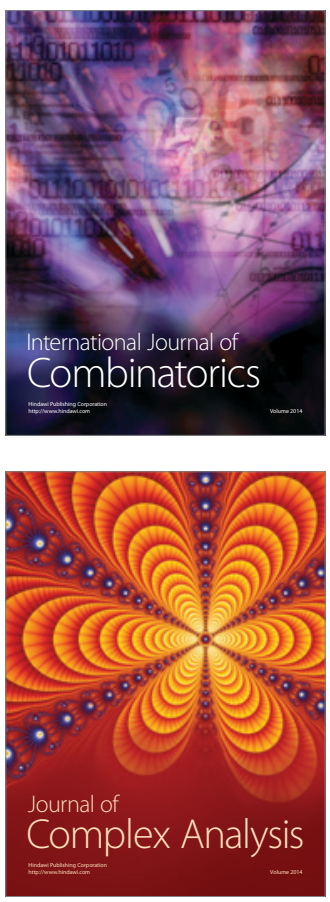

International Journal of

Mathematics and

Mathematical

Sciences
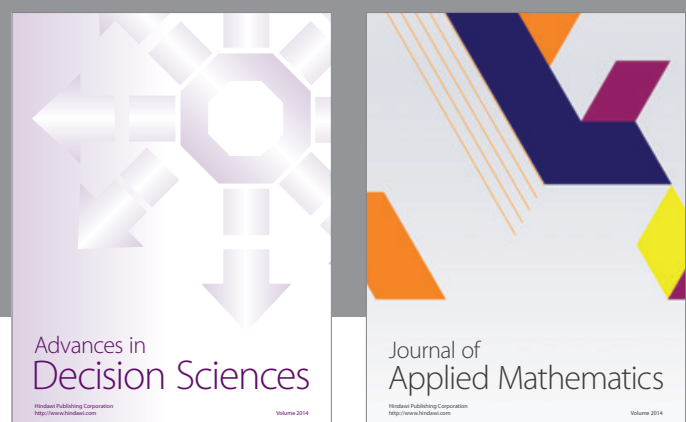

Journal of

Applied Mathematics
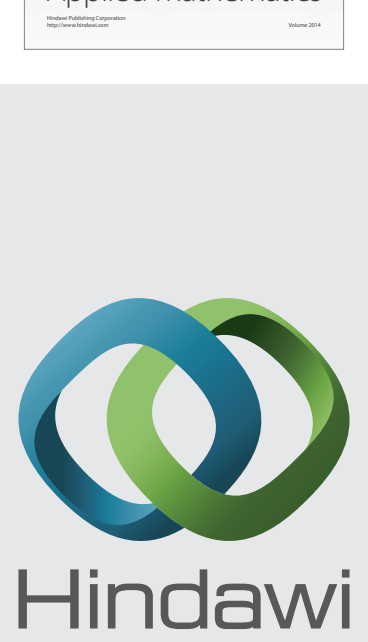

Submit your manuscripts at http://www.hindawi.com
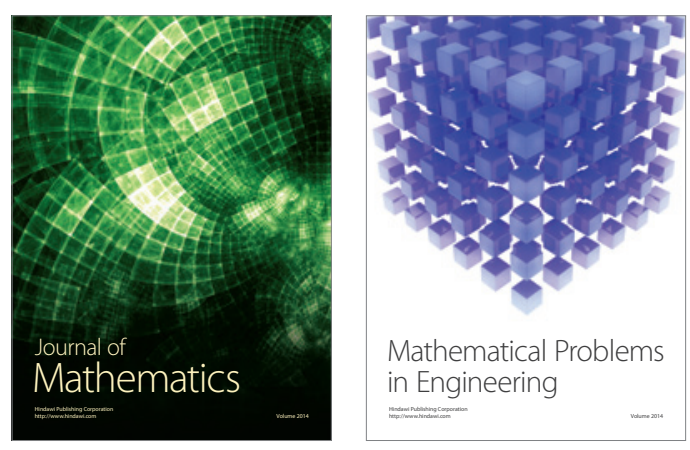

Mathematical Problems in Engineering
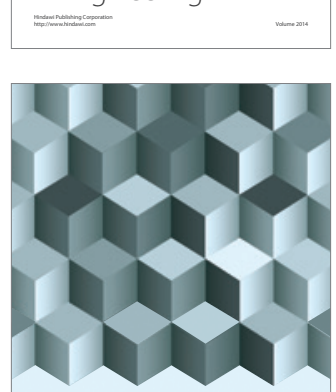

Journal of

Function Spaces
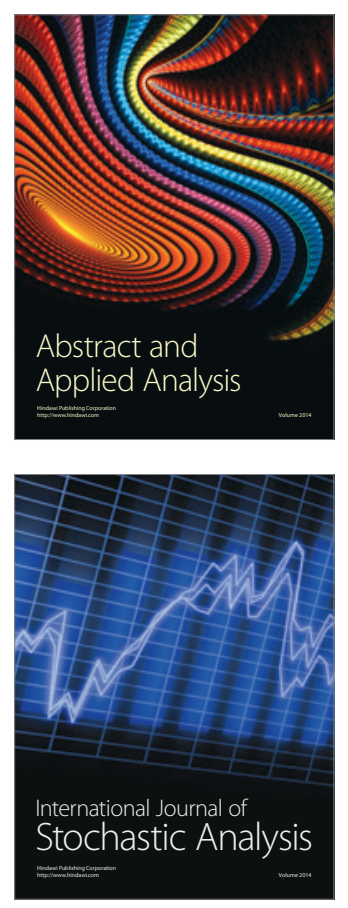

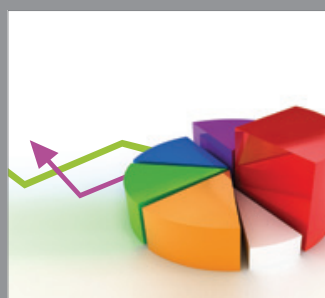

ournal of

Probability and Statistics

Promensencen
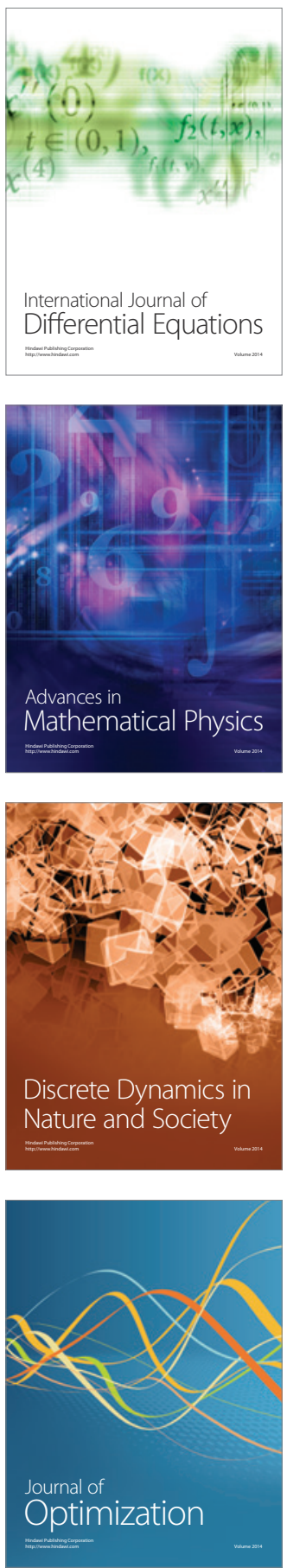